\title{
Different numerical techniques for the solution of transcendental eigenvalue problem in transmission line bundles
}

\author{
Himanshu Verma* Peter Hagedorn*
}

(Received 24 April 2006; revised 13 May 2007)

\begin{abstract}
The problem of a bundled transmission line attached with many spacer dampers is formulated by considering the conductor as a continuous system. The formulation results in a complex transcendental eigenvalue problem (TEVP), which presents several numerical difficulties. Some approaches towards solving the TEVP and their respective difficulties in getting the solutions are discussed in detail. The solution of the TEVP gives the necessary input data for applying the energy balance, which is used in order to obtain the actual vibration amplitudes of the transmission line conductors.
\end{abstract}

*Dynamics and Vibrations Group, Department of Mechanical Engineering, Technische Universität Darmstadt, Hochschulstraße-1, D-64289, Darmstadt, Germany.

mailto:verma@dyn.tu-darmstadt.de

See http://anziamj.austms.org.au/V47EMAC2005/Verma for this article, (c) Austral. Mathematical Soc. 2007. Published July 20, 2007. ISSN 1446-8735 


\section{Contents}

1 Introduction

C874

2 Modeling of a bundled conductor system

C877

2.1 Basic configuration . . . . . . . . . . . . . C877

2.2 Modeling the conductor and the boundary conditions . . . C877

2.3 Incorporating the spacer damper into model . . . . . . . . C880

3 Solution of the eigenvalue problem

C885

3.1 Determinant search method . . . . . . . . . . . C885

3.2 Alternate approach . . . . . . . . . . . . . C C886

3.3 Continuous spectrum approach . . . . . . . . . C C887

4 Results

C888

5 Conclusions

C890

References

C891

\section{Introduction}

In high voltage power transmission lines the use of bundled conductors is very common. A conductor bundle consists of multiple (two to eight) subconductors. It is necessary to maintain a certain distance between the subconductors for electrical reasons. Spacers at regular intervals of approximately $40 \mathrm{~m}$ to $50 \mathrm{~m}$ are used for this purpose. Bending strains produced at the spacer clamps due to aeolian vibrations, which take place in the frequency range of 3 to $50 \mathrm{~Hz}$ [1], are not desirable. Therefore, the joints between spacer arms and spacer frame are made of resilient material so as to damp out the vibrations. Such a spacer is named a spacer damper. Figure 1 shows spacer dampers corresponding to different number of sub-conductors. 


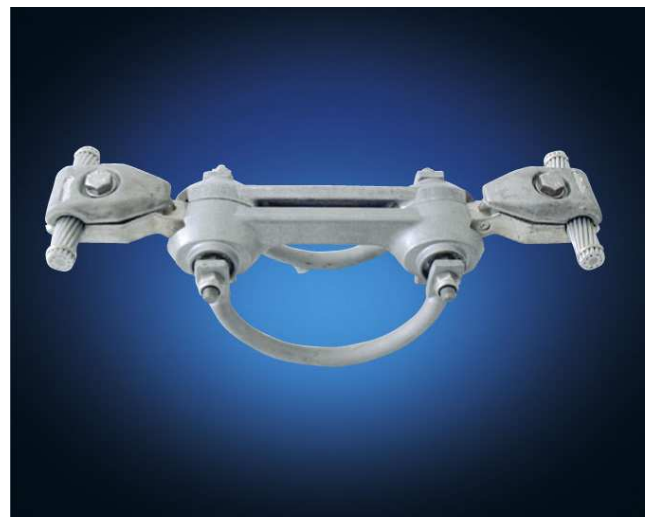

(a)

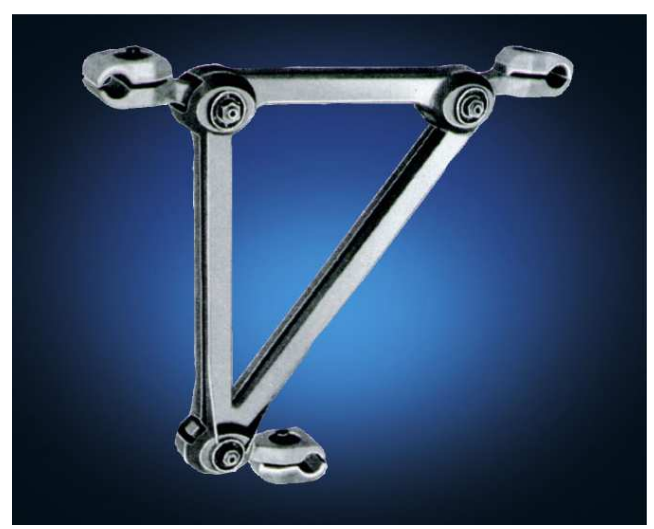

(b)

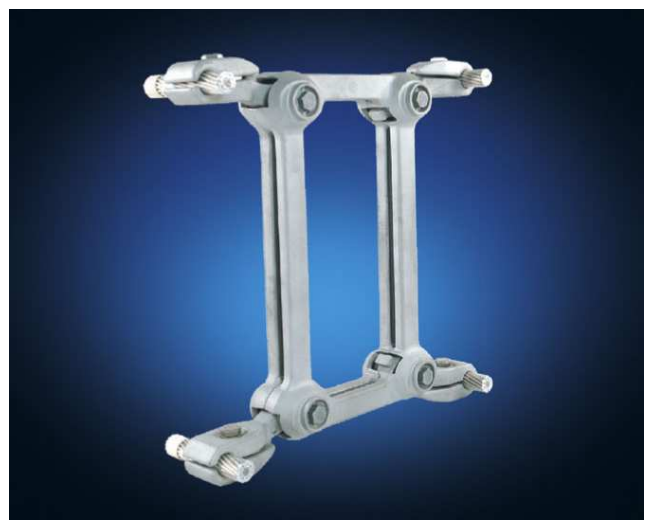

(c)

Figure 1: Typical spacer dampers (courtesy of Ribe Electrical Fittings GmbH): (a) Twin bundle; (b) Triple bundle; and (c) Quad bundle. 
Mathematical models are necessary for the computation of vibrations of bundled conductor attached with many spacer dampers, for studying the efficiency of damping measures. The energy balance principle $[2,3]$ is well established for estimating the vibrations of single conductor transmission lines. However, modifications are needed to use it for bundled conductors. In the standard form of the energy balance principle, the complex eigenvalue problem (EVP) is solved first, to get system frequencies and the corresponding mode shapes. In the second step, the energy input from the wind is equated with the energy dissipated due to conductor's self damping and that due to external damping devices (for example, Stockbridge damper and spacer damper) in order to get the actual vibration amplitudes for each mode.

Modeling of transmission line as a continuous system leads to a transcendental system matrix, having infinitely many eigenvalues. For applying the energy balance principle, one has to obtain eigenvalues of the system matrix in the frequency range of interest (that is, 3 to $50 \mathrm{~Hz}$ for aeolian vibrations). In the case of bundled conductors attached with spacer dampers, the system matrix shows poor numerical behavior. Thus, many eigenvalues are missed when conventional methods are used to solve this transcendental EvP. The prime reason for poor numerical behavior of the system matrix is the large range in the order of magnitude of its elements.

We describe different methods for obtaining the solution of the transcendental EvP for bundled conductors. The problem of bundled conductors with many spacer dampers is formulated first. The form of the system matrix shows the reasons behind its poor numerical behavior. Different methods for solving this transcendental EVP are discussed. A new method, named Continuous Spectrum Approach, has been developed for getting an engineering solution of such EVP for bundled conductors. One representative example problem is solved for the validation of this approach. Note that besides vortex excited oscillations, many other types of vibrations are possible in bundled conductors. However, they are not the subject of this article. 


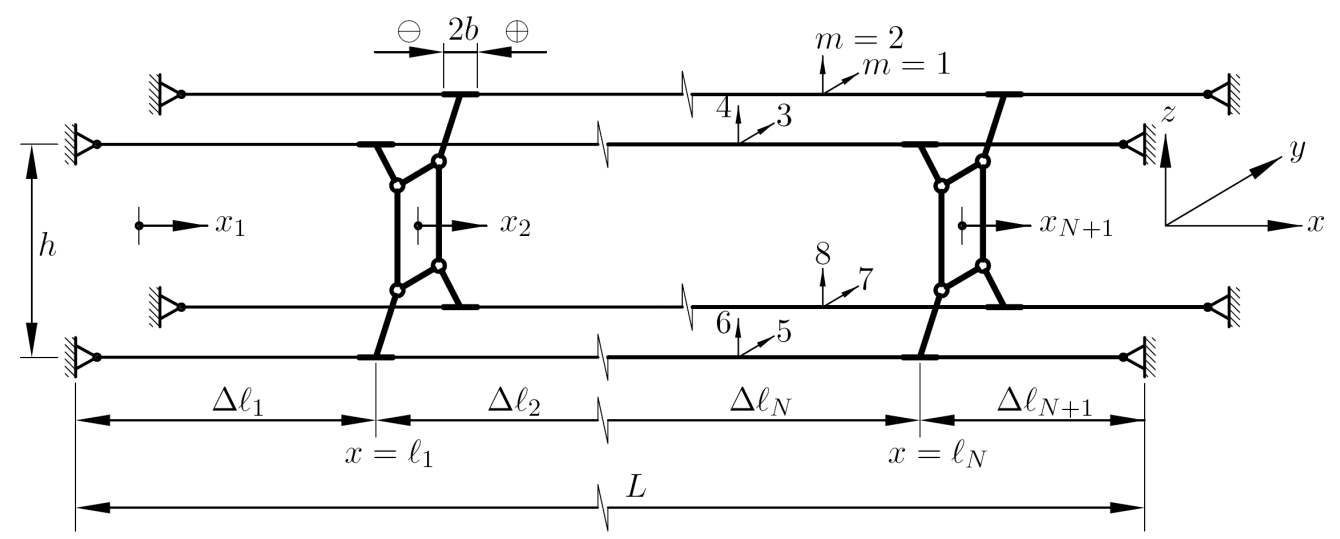

FiguRE 2: Schematic diagram of quad bundle attached with $N$ similar spacer dampers

\section{Modeling of a bundled conductor system}

\subsection{Basic configuration}

Consider a conductor bundle consisting of $M$ subconductors. In the current work $M$ is taken as four, and hence the quad spacer dampers will be considered for the modelling. $N$ similar spacer dampers are attached to it as shown in Figure 2. Each spacer damper has $M$ arms. The spacer dampers are placed at $x=l_{n}$ where $n=1,2,3, \ldots, N$, from the left end. The spacers divide the total span of length $L$, into $N+1$ subspans of corresponding lengths, $\Delta l_{1}, \Delta l_{2}, \ldots, \Delta l_{N+1}$.

\subsection{Modeling the conductor and the boundary conditions}

We make the following assumptions to simplify the formulation: 


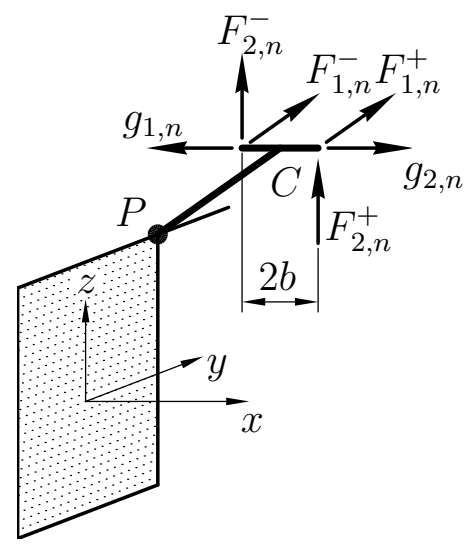

FiguRE 3: Forces acting at the clamp of a spacer damper

1. The sag to span ratio is small so that the sag can be neglected.

2. The bending stiffness $E I$ of the conductor is disregarded because of $\sqrt{E I / T} \ll \lambda /(2 \pi)$. The conductor is considered as a string.

3. The conductors are assumed to be clamped at the span ends.

4. The bundle vibrates in one of its eigenmodes and exhibits synchronous motion.

Although the excitation due to aeolian vibration is prominently in the vertical direction, the presence of spacer dampers complicates the conductor motion. Hence, transverse displacements of conductor in both, the vertical and the horizontal directions have to be considered. The transverse motion of the conductor in the $v$ th subspan $(v=1,2,3, \ldots, N+1)$ is expressed by the wave equation

$$
\rho A \ddot{w}_{m, v}(x, t)-T_{m} w_{m, v}^{\prime \prime}(x, t)=0, \quad m=1,2,3, \ldots, 2 M,
$$

where $\rho A$ is the unit mass of the conductor, $T_{m}$ is the conductor tension and $m$ represents the plane of motion of the conductor. $w_{m, v}(x, t)$ represents the transverse displacement of the conductor in $m$ th direction and in 
$v$ th subspan, at a location $x$ and a time instant $t$. In (1) and in all coming equations (') represents the derivative with respect to time and ( )' represents the derivative of a quantity with respect to space. Odd values of $m$ correspond to the horizontal displacement and even values correspond to the vertical displacements (refer to Figure 2). Since the conductors are clamped at both ends, the displacements are zero at the supports, that is,

$$
\begin{aligned}
w_{m, 1}(0, t) & =0, \\
w_{m, N+1}(L, t) & =0 .
\end{aligned}
$$

Equations (1-3) define a boundary value problem. For the sake of convenience, we consider new subspan coordinates $x_{v}$, which are defined as

$$
x_{v}=x-l_{v-1}, \quad 0<x_{v}<\Delta l_{v},
$$

with $l_{0}=0$. By separating the space and time variables as

$$
w_{m, v}\left(x_{v}, t\right)=W_{m, v}\left(x_{v}\right) \exp s t
$$

and substituting in Equation (1), we get the solution in the form

$$
W_{m, v}\left(x_{v}\right)=A_{m, v} \exp \left(\frac{s x_{v}}{c_{m}}\right)+B_{m, v} \exp \left(\frac{-s x_{v}}{c_{m}}\right)
$$

where $A_{m, v}$ and $B_{m, v}$ are integration constants, and $c_{m}=\sqrt{T_{m} / \rho A}$ is the wave velocity in $m$ th direction. Substituting this solution in the boundary conditions $(2,3)$ gives

$$
\begin{aligned}
A_{m, 1}+B_{m, 1} & =0, \\
A_{m, N+1} \exp \left(\frac{s \Delta l_{N+1}}{c_{m}}\right)+B_{m, N+1} \exp \left(\frac{-s \Delta l_{N+1}}{c_{m}}\right) & =0 .
\end{aligned}
$$




\subsection{Incorporating the spacer damper into model}

Figure 3 represents the $n$th spacer damper of Figure 2 with the forces acting at the clamp. The Effective clamp width $2 b$ is the sum of the physical clamp width and twice of the characteristic length $l_{\text {char }}$ of the conductor due to bending (where $l_{\text {char }}=\sqrt{E I / T}[2,3]$ ). In this figure only one of the four arms is shown attached to the central frame via a viscoelastic joint at the point $P$. The forces in the directions transverse to the conductor are denoted by $F_{m, n}^{-}$or $F_{m, n+1}^{+}, m=1,2,3, \ldots, 2 M$ and $n=1,2,3, \ldots, N$. Here, ()$^{+}$ and ()$^{-}$show whether the point of interest is in positive or in negative $x$-direction. The forces in the axial direction of the conductor are denoted by $g_{j, n}, j=1,2$ and $n=1,2,3, \ldots, N$.

The force and velocity vector at the $n$th spacer damper, as shown in Figure 3, are

$$
\mathbf{F}_{n}(t)=\left[\begin{array}{c}
F_{1, n}^{-} \\
F_{2, n}^{-} \\
F_{1, n+1}^{+} \\
F_{2, n+1}^{+} \\
F_{3, n}^{-+} \\
F_{4, n}^{-} \\
\vdots \\
F_{2 M-1, n+1}^{+} \\
F_{2 M, n+1}^{+}
\end{array}\right], \quad \dot{\mathbf{w}}_{n}(t)=\left[\begin{array}{c}
\dot{w}_{1, n}^{-}\left(\Delta l_{n}-b, t\right) \\
\dot{w}_{2, n}^{-}\left(\Delta l_{n}-b, t\right) \\
\dot{w}_{1, n+1}^{+}(b, t) \\
\dot{w}_{2, n+1}^{+}(b, t) \\
\dot{w}_{3, n}^{-}\left(\Delta l_{n}-b, t\right) \\
\dot{w}_{4, n}^{-}\left(\Delta l_{n}-b, t\right) \\
\vdots \\
\dot{w}_{2 M-1, n+1}^{+}(b, t) \\
\dot{w}_{2 M, n+1}^{+}(b, t)
\end{array}\right]
$$

and they are expressed as

$$
\mathbf{F}_{n}(t)=\Re\left[\widehat{\mathbf{F}}_{n} \exp s t\right], \quad \dot{\mathbf{w}}_{n}(t)=\Re\left[\widehat{\dot{\mathbf{w}}}_{n} \exp s t\right] .
$$

Here [ ${ }^{\wedge}$ ] shows the amplitude of the respective quantity and $b$ is sum of half the physical clamp width of the spacer damper and the characteristic length of the conductor due to bending. In modeling of bundled conductors, 
the effect of spacer dampers is incorporated by mean of their impedance matrices $\mathbf{Z}(s)$, which are obtained by considering the mass, geometric and damping properties of the spacer dampers [4]. In doing so, it is treated as a multi-body system with linear elastic joints (for example, four arms and the central frame of a quad spacer are treated as five interconnected rigid bodies). The formulation results in a square impedance matrix, elements of which are functions of the frequency of conductor vibration [4]. The impedance matrix $\mathbf{Z}(s)$, is of size $4 M \times 4 M$, which relates the $4 M$ velocities at the clamps to the $4 M$ transverse forces acting at the clamps. Four transverse forces acting at each clamp can be seen in Figure 3. The amplitudes $\widehat{\mathbf{F}}_{n}$ and $\widehat{\dot{\mathbf{w}}}_{n}$ are related to each other through

$$
\widehat{\mathbf{F}}_{n}=\mathbf{Z}(s) \widehat{\dot{\mathbf{w}}}_{n}, \quad n=1,2,3, \ldots, N .
$$

Anderson \& Hagedorn [4] describe the process of obtaining $\mathbf{Z}(s)$, which will be directly adopted here and $\mathbf{Z}(s)$ is assumed to be known beforehand in this article. The forces at the clamp ends of any spacer damper will be in equilibrium with the transverse components of tensions on respective sides of the clamp

$$
\begin{aligned}
F_{m, n}^{-}(t) & =-T_{m} w_{m, n}^{\prime}\left(\Delta l_{n}-b, t\right), \\
F_{m, n+1}^{+}(t) & =T_{m} w_{m, n+1}^{\prime}(b, t) .
\end{aligned}
$$

Substituting for $w_{m, n}$ from Equation (5) and (6) gives

$$
\begin{aligned}
\widehat{F}_{m, n}^{-}= & -\frac{T_{m} s}{c_{m}}\left[A_{m, n} \exp \left(\frac{s \Delta l_{n}}{c_{m}}\right) \exp \left(\frac{-s b}{c_{m}}\right)\right. \\
& \left.-B_{m, n} \exp \left(\frac{-s \Delta l_{n}}{c_{m}}\right) \exp \left(\frac{s b}{c_{m}}\right)\right], \\
\widehat{F}_{m, n+1}^{+}= & \frac{T_{m} s}{c_{m}}\left[A_{m, n} \exp \left(\frac{s b}{c_{m}}\right)-B_{m, n} \exp \left(\frac{-s b}{c_{m}}\right)\right] .
\end{aligned}
$$


Taking $\xi_{m}^{ \pm}(s)=\exp \left( \pm \frac{s b}{c_{m}}\right), \chi_{m, n}^{ \pm}=\exp \left( \pm \frac{s \Delta l_{n}}{c_{m}}\right)$, the expressions for forces and velocities at the clamps are

$$
\begin{aligned}
\widehat{F}_{m, n}^{-} & =-\frac{T_{m} s}{c_{m}}\left[A_{m, n} \chi_{m, n}^{+} \xi_{m}^{-}-B_{m, n} \chi_{m, n}^{-} \xi_{m}^{+}\right], \\
\widehat{F}_{m, n+1}^{+} & =\frac{T_{m} s}{c_{m}}\left[A_{m, n+1} \xi_{m}^{+}-B_{m, n+1} \xi_{m}^{-}\right],
\end{aligned}
$$

and

$$
\begin{aligned}
& \left.\widehat{\dot{w}}_{m, n}\right|_{x_{n}=\Delta l_{n}-b}=s\left(A_{m, n} \chi_{m, n}^{+} \xi_{m}^{-}+B_{m, n} \chi_{m, n}^{-} \xi_{m}^{+}\right), \\
& \left.\widehat{\dot{w}}_{m, n+1}\right|_{x_{n+1}=b}=s\left(A_{m, n+1} \xi_{m}^{+}+B_{m, n+1} \xi_{m}^{-}\right) .
\end{aligned}
$$

Equations $(16,17)$ express the forces as a product of tension and slope, and Equation (11) expresses them as a product of the velocity and the impedance. Equating these two set of equations gives us $4 M N$ equations as explained below. For explanation we consider only the first element of the force vector from Equation (11),

$$
\widehat{F}_{1, n}^{-}=\left\{\begin{array}{llll}
Z_{1,1} & Z_{1,2} & \ldots & Z_{1,4 M}
\end{array}\right\} \widehat{\dot{\mathbf{w}}}_{n},
$$

the same element of the force vector from Equation (16) is

$$
\widehat{F}_{1, n}^{-}=-\frac{T_{1} s}{c_{1}}\left(A_{1, n} \chi_{1, n}^{+} \xi_{1}^{-}-B_{1, n} \chi_{1, n}^{-} \xi_{1}^{+}\right) .
$$

Equating the right hand sides of Equations $(20,21)$ and rearranging gives

$$
\begin{aligned}
0= & A_{1, n} \chi_{1, n}^{+} \xi_{1}^{-}\left(Z_{1,1}+\frac{T_{1}}{c_{1}}\right)+B_{1, n} \chi_{1, n}^{-} \xi_{1}^{+}\left(Z_{1,1}-\frac{T_{1}}{c_{1}}\right) \\
& +\left(A_{2, n} \chi_{2, n}^{+} \xi_{2}^{-}+B_{2, n} \chi_{2, n}^{-} \xi_{1}^{+}\right) Z_{1,2} \\
& +\left(A_{1, n+1} \chi_{1, n+1}^{+} \xi_{1}^{-}+B_{1, n+1} \chi_{1, n+1}^{-} \xi_{1}^{+}\right) Z_{1,3} \\
& +\left(A_{2, n+1} \chi_{2, n+1}^{+} \xi_{2}^{-}+B_{2, n+1} \chi_{2, n+1}^{-} \xi_{1}^{+}\right) Z_{1,4}
\end{aligned}
$$




$$
\begin{aligned}
& \quad \vdots \\
& +\left(A_{2 M, n+1} \chi_{2 M, n+1}^{+} \xi_{2 M}^{-}+B_{2 M, n+1} \chi_{2 M . n+1}^{-} \xi_{2 M}^{+}\right) Z_{1,4 M} .
\end{aligned}
$$

Corresponding to each spacer we will have $4 M$ such equations. These $4 M N$ equations for $N$ spacers and the $4 M$ equations from boundary conditions at the span ends in $(7,8)$ result in a transcendental EVP:

$$
\mathbf{J}(s) \mathbf{a}=\mathbf{0},
$$

where

$$
\begin{aligned}
& \mathbf{a}=\left\langle A_{1,1}, B_{1,1}, A_{2,1}, B_{2,1}, \ldots, A_{2 M, 1}, B_{2 M, 1}, \ldots,\right. \\
& \quad A_{1, v}, B_{1, v}, A_{2, v}, B_{2, v}, \ldots, A_{2 M, v}, B_{2 M, v}, \ldots, \\
& \left.\quad A_{1, N+1}, B_{1, N+1}, A_{2, N+1}, B_{2, N+1}, \ldots, A_{2 M, N+1}, B_{2 M, N+1}\right\rangle^{T}
\end{aligned}
$$

and the matrix

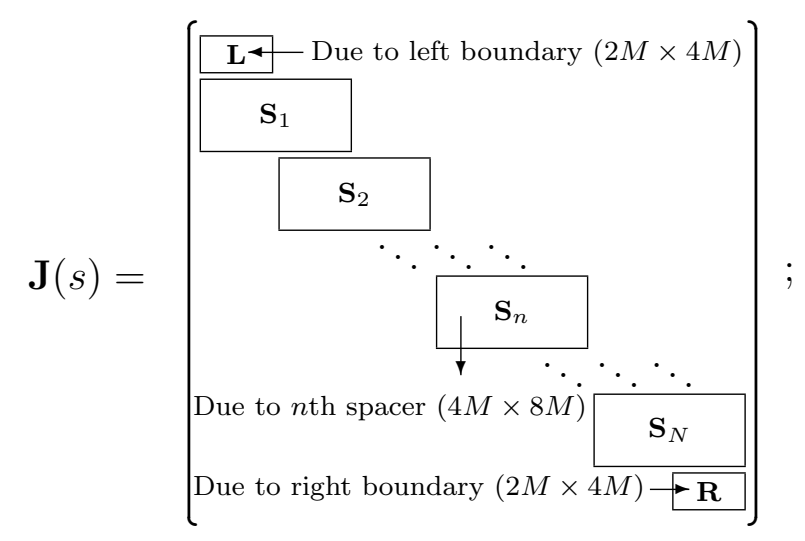

where $\mathbf{L}$ and $\mathbf{R}$ are the matrices of size $2 M \times 4 M$ and $\mathbf{S}_{1}, \mathbf{S}_{2}, \ldots, \mathbf{S}_{n}$ are of size $4 M \times 8 M$. The elements of matrices $\mathbf{L}, \mathbf{S}_{n}$ and $\mathbf{R}$ are shown below. The 
matrix $\mathbf{L}$ is due to left homogeneous boundary conditions

$$
\mathbf{L}=\left[\begin{array}{ccccccccc}
1 & 1 & 0 & 0 & \ldots & 0 & 0 & 0 & 0 \\
1 & 1 & 0 & 0 & \ldots & 0 & 0 & 0 & 0 \\
0 & 0 & 1 & 1 & \ldots & 0 & 0 & 0 & 0 \\
\vdots & \vdots & \vdots & \vdots & \ddots & \vdots & \vdots & \vdots & \vdots \\
0 & 0 & 0 & 0 & \ldots & 1 & 1 & 0 & 0 \\
0 & 0 & 0 & 0 & \ldots & 0 & 0 & 1 & 1
\end{array}\right]
$$

matrix $\mathbf{S}_{n}$ is a complex matrix due to $n$th spacer damper:

$$
\begin{aligned}
& \mathbf{S}_{n}=\left[\mathbf{P}_{1, n} \mathbf{P}_{2, n} \cdots \mathbf{P}_{p, n} \cdots \mathbf{Q}_{3, n} \mathbf{Q}_{4, n} \cdots \mathbf{Q}_{q, n} \cdots\right], \\
& \text { for all } p=1,2,5,6,9,10, \ldots,(4 M-3),(4 M-2), \\
& \text { for all } q=3,4,7,8,11,12, \ldots,(4 M-1), 4 M,
\end{aligned}
$$

where

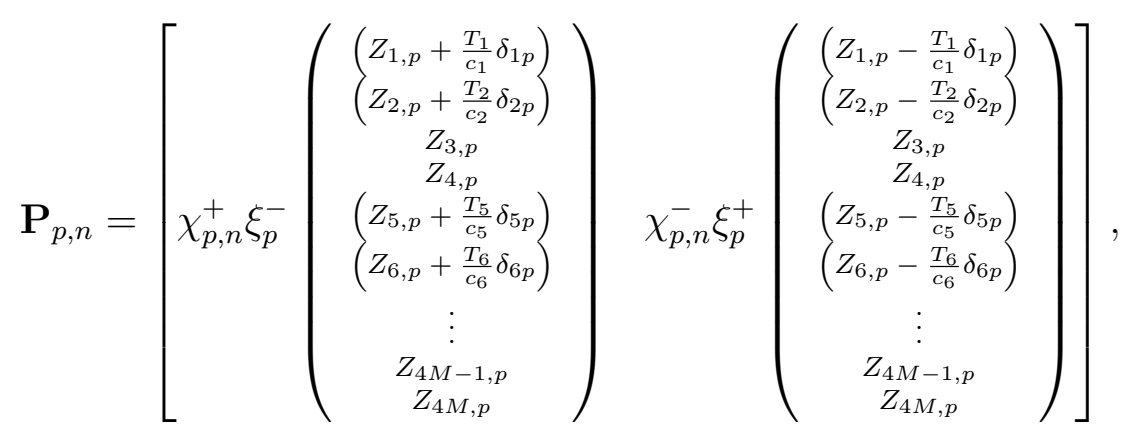

and

$$
\mathbf{Q}_{q, n}=\left[\xi_{q}^{+}\left(\begin{array}{c}
Z_{1, q} \\
Z_{2, q} \\
\left(Z_{3, q}-\frac{T_{3}}{c_{3}} \delta_{3 q}\right) \\
\left(Z_{4, q}-\frac{T_{4}}{c_{4}} \delta_{4 q}\right) \\
Z_{5, q} \\
Z_{6, q} \\
\vdots \\
\vdots \\
\left(Z_{4 M-1, q}-\frac{T_{4 M-1}}{c_{4 M-1}} \delta_{(4 M-1) q}\right) \\
\left(Z_{4 M, q}-\frac{T_{4 M} M}{c_{4 M}} \delta_{(4 M) q}\right)
\end{array}\right) \quad \xi_{q}^{-}\left(\begin{array}{c}
Z_{1, q} \\
Z_{2, q} \\
\left(Z_{3, q}+\frac{T_{3}}{c_{3}} \delta_{3 q}\right) \\
\left(Z_{4, q}+\frac{T_{4}}{c_{4}} \delta_{4 q}\right) \\
Z_{5, q} \\
Z_{6, q} \\
\vdots \\
\left(Z_{4 M-1, q}+\frac{T_{4 M-1}}{c_{4 M-1}} \delta_{(4 M-1) q}\right) \\
\left(Z_{4 M, q}+\frac{T_{4 M}}{c_{4 M}} \delta_{(4 M) q}\right)
\end{array}\right)\right]
$$


Matrix $\mathbf{R}$ is due to right hand homogeneous boundary conditions and again is a complex matrix:

$$
\mathbf{R}=\left[\begin{array}{ccccccccc}
\chi_{1, n+1}^{+} & \chi_{1, n+1}^{-} & 0 & 0 & \ldots & 0 & 0 & 0 & 0 \\
0 & 0 & \chi_{2, n+1}^{+} & \chi_{2, n+1}^{-} & \cdots & 0 & 0 & 0 & 0 \\
\vdots & \vdots & \vdots & \vdots & \ddots & \vdots & \vdots & \vdots & \vdots \\
0 & 0 & 0 & 0 & \cdots & \chi_{2 M-1, n+1}^{+} & \chi_{2 M-1, n+1}^{-} & 0 & 0 \\
0 & 0 & 0 & 0 & \cdots & 0 & 0 & \chi_{2 M, n+1}^{+} & \chi_{2 M, n+1}^{-}
\end{array}\right]
$$

\section{Solution of the eigenvalue problem}

The matrix equation (23) constitutes an EVP having infinitely many eigenvalues. Since the matrix $\mathbf{J}(s)$ contains system parameters it is referred to as the system matrix. The elements of the system matrix are transcendental functions of the parameter $s$, which is a complex number $(s=-\delta+\mathrm{i} \omega)$ as the system is damped. The imaginary part of $s$ represents a circular frequency and the real part represents a decay coefficient. From its constitution, the elements of the system matrix have a large variation in magnitude, which makes it behave numerically poor. We faced difficulties in finding all of the system eigenvalues, in the frequency range of consideration (that is, 3 to $50 \mathrm{~Hz}$ ) by conventional methods of solving transcendental EVP's [5, 6, 7, 8, 9, 10, 11, 12]. In what follows, different approaches are discussed for solving this numerically poor transcendental eigenvalue problem.

\subsection{Determinant search method}

One technique for solving the transcendental EvP is by the determinant search method [13,3], in which one searches values of $s$ for which

$$
\operatorname{det}[\mathbf{J}(s)]=0 .
$$


Since the parameter $s$ is a complex number, the search domain is two dimensional. Finding all of the eigenvalues with this approach is only possible for relatively small systems. Due to the poor numerical behavior of the system matrix $\mathbf{J}(s)$, it is easy to miss many values of $s$ for which the determinant of $\mathbf{J}(s)$ is zero. Although normalization of the basic variables leads to a better conditioned system matrix, difficulties are faced when trying to find the eigenvalues for big system matrices with the determinant search method. The major difficulty one faces is that, for a big system matrix the optimizer does not converge to zero, rather it sticks to a minimum. The prime reason for this difficulty is the large variation in the order of numerical values of the elements of the system matrix, which, together with the large number of algebraic operations involved in calculating the determinant, lead to such numerical problems. Moreover, additional checks are required to confirm whether the obtained values are eigenvalues of the system or not.

\subsection{Alternate approach}

To overcome the difficulties faced in the determinant search method, we used an alternate approach in which the homogeneous set of $4 M(N+1)$ simultaneous equations, given by Equation (23), is first transformed into a non-homogeneous system by using any $\{4 M(N+1)-1\}$ equations. A simple and better conditioned optimization criterion is then defined for the remaining equation. The steps are described in more details by the following.

\section{Let $A_{1,1}=1$.}

2. Substitute the value of $A_{1,1}$ into any $\{4 M(N+1)-1\}$ homogeneous equations of Equation (23), so as to transform them into a set of nonhomogeneous equations. Determine other system variables, that is, $B_{1,1}, A_{2,1}, B_{2,1}, \ldots, A_{2 M, N+1}, B_{2 M, N+1}$ (refer Equation (24)), by solving these $\{4 M(N+1)-1\}$ non-homogeneous equations with the help of Gauss elimination. 
3. Substitute the values of $B_{1,1}, A_{2,1}, B_{2,1}, \ldots, A_{2 M, N+1}, B_{2 M, N+1}$ back into remaining one [say $l$ th] equation. Since the perviously assumed $A_{1,1}$ was not the correct one, these values will not satisfy the $l$ th equation and will result in an error $\varepsilon$ :

$$
\begin{aligned}
& J_{l, 1}+B_{1,1} J_{l, 2}+A_{2,1} J_{l, 3}+B_{2,2} J_{l, 4}+\cdots \\
& +A_{2 M, N+1} J_{l,\{4 M(N+1)-1\}}+B_{2 M, N+1} J_{l, 4 M(N+1)}=\varepsilon .
\end{aligned}
$$

The optimization criterion, now, is to search such values of $s$, in the complex domain, for which $|\varepsilon|$ equals (or tends to) zero. This approach leads to a numerically well behaving optimization criterion, which converges faster than the determinant search method. It gives most, if not all, of the eigenvalues provided we give a good initial guess. Moreover, one can always be sure that the value of $s$ found after minimizing $\varepsilon$, is an eigenvalue of the system in Equation (23). However, this approach requires good initial guesses, otherwise it fails to find very closely spaced eigenvalues because of the two dimensional search domain.

\subsection{Continuous spectrum approach}

We observed that in the case of very big system matrices (for example, bundled conductors attached with many spacer dampers), the aforementioned approaches fail to find some of the eigenvalues, even if the given initial guesses are good enough. A plausible reason for missing eigenvalues is the two dimensional search domain. Specially in the case of bundled conductors the system frequencies are very closely spaced (at $0.001 \mathrm{~Hz}$ ). If two parameters (that is, $\delta$ and $\omega$ ) are simultaneously searched, it is very likely that the optimizer will stick to one combination of these parameters for many consecutive guess values. It poses a major difficulty of missing the eigenvalues even with good initial guesses.

In the continuous spectrum approach the dense frequency spectrum of the 
bundled conductors is utilized to reduce the search domain from two dimensional to one dimensional. Since for a bundled conductor the spacing of the eigenfrequencies is very small, it can be assumed to have a continuous frequency spectrum [2]. Thus it is reasonable to assume that every frequency, in the range of consideration, is a natural frequency of the system. This means that we know the complex parts $\omega$ of eigenvalues $s$ beforehand. Corresponding to each $\omega$ value there exist one $\delta$ value which satisfies

$$
\mathbf{J}(\delta) \mathbf{a}=\mathbf{0} .
$$

Therefore, the criterion now is to find the corresponding $\delta$ value for closely spaced $\omega$ values, using any of the above two approaches. This simplification converts our two dimensional optimization problem (in the complex domain) to one dimensional (in the real domain). The results section shows the peaks in the continuous plot represent the eigenvalues of the system. In the current work the entire coding was done in MATLAB 6.5, and for minimizing $\varepsilon$, among others, the in-built function $\mathrm{f}$ solve was used.

After finding the eigenvalues $s$ of the system matrix, the corresponding eigenvectors were found by solving an equivalent algebraic matrix EVP of the form

$$
[\mathbf{J}(s)-\lambda \mathbf{I}] \mathbf{a}=\mathbf{0} .
$$

The eigenvector corresponding to any eigenvalue $s$ of the transcendental EVP of Equation (23) is obtained by finding the eigenvector associated to $\lambda=0$ in the equivalent algebraic EVP of Equation (33) [3].

\section{Results}

To compare the effectiveness of the described approaches, a representative example problem of a bundled conductor was considered (refer Figure 2). A quad bundled transmission line (that is, $M=4$ ) of span length $L=140 \mathrm{~m}$ 


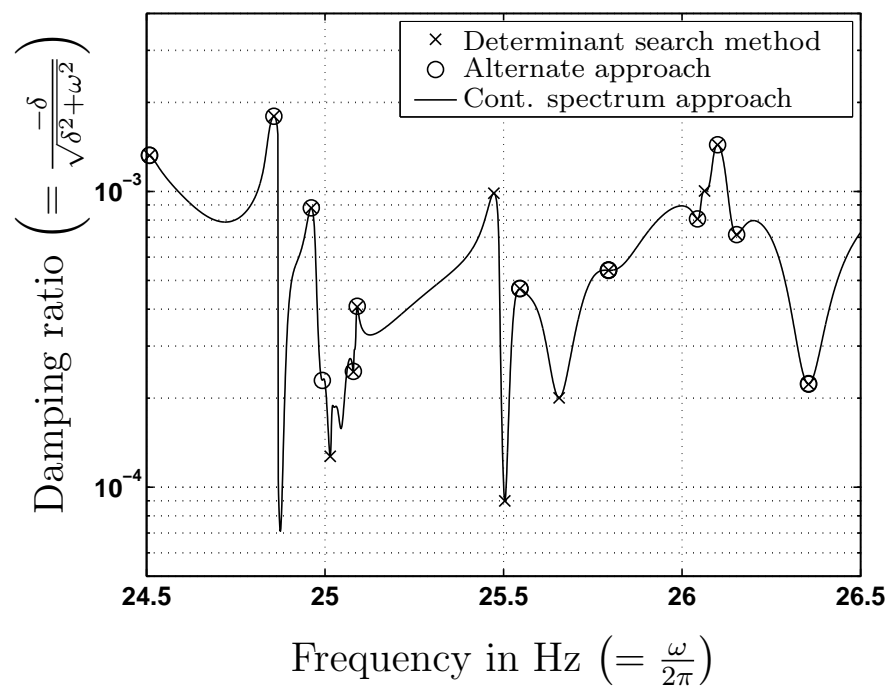

FiguRE 4: Obtained frequencies from different methods $(24.5-26.5 \mathrm{~Hz})$

has been taken. It carries two quad spacer dampers, which are attached respectively at $l_{1}=40 \mathrm{~m}$ and $l_{2}=90 \mathrm{~m}$, from the left end. Both the spacer dampers are taken of the same mass, geometric and damping characteristics. The diameter of the conductor is $17.5 \mathrm{~mm}$ and its mass per unit length is $0.981 \mathrm{~kg} / \mathrm{m}$. The tension in all four conductors is $50 \mathrm{kN}$.

The transcendental EVP was solved with the above described approaches and the eigenvalues were searched in the frequency range of 20 to $30 \mathrm{~Hz}$. Figure 4 compare the obtained eigenvalues from these three approaches. In the figures, the eigenfrequencies in Hertz have been plotted with respect to their corresponding damping ratios. Because of their small values, the damping ratios have been plotted on the log-scale. Figure 4 shows that the system eigenfrequencies are very closely spaced. For a better illustration, eigenvalues in the frequency range of 24.5 to $26.5 \mathrm{~Hz}$ have only been plotted; however the frequency range of interest lies between 3 to $50 \mathrm{~Hz}$ [1]. 
These figures show that there are many eigenvalues which were either missed by the determinant search method or by the alternate approach. However, the continuous curve, which has been obtained by the continuous spectrum approach, passes through all the eigenvalues found from the other two approaches. We observed that all the peaks in the continuous curve represent the system eigenvalues, as seen in Figure 4. Thus one can easily identify that the continuous spectrum approach is able to search many extra eigenvalues, which were missed by the other two approaches (refer Figure 4).

The continuous spectrum approach provides us with a continuous curve of damping ratios corresponding to all the frequencies in the range of interest. For a transmission line engineer the major interest is in knowing the maximum strains levels in transmission line, corresponding to each eigenvalue. Strains can now be computed after knowing the mode shapes and the actual vibration amplitudes, which are obtained by the energy balance principle.

\section{Conclusions}

The problem of aeolian vibrations of bundled conductors attached with many spacer dampers was formulated first. The formulation resulted in a numerically poor transcendental system matrix. Thus obtained system matrix posed numerical problems in the solution of transcendental EVP because of its bigger size and large differences in the order of numerical values of its elements. The classical determinant search method and one alternate approach were discussed for solving the transcendental EVP, which Verma \& Hagedorn [3] also used for the solution of simpler problem of single conductor transmission lines. A new approach, named as continuous spectrum approach, was presented for the solution of EVP in the case of bundled conductors. The new approach provides a good engineering solution for the transcendental EVP. One representative example problem was solved by all described approaches and the results were compared. 
Acknowledgements: The authors acknowledge the support given by RIBE Elektroarmaturen GmbH \& Co., K. G. Schwabach, Ministry of Hessen and DFG (German Research Foundation).

\section{References}

[1] EPRI. Transmission Line Reference Book, Wind Induced Conductor Motion. Palo Alto, California: Electrical Power Research Institute, 1979. C874, C889

[2] P. Hagedorn. On the Computation of Damped Wind Excited Vibrations of Overhead Transmission Lines. Journal of Sound and Vibration, 83(2):253-271, 1982. doi:10.1016/S0022-460X(82)80090-4 C876, C880, C888

[3] H. Verma and P. Hagedorn. Wind induced vibrations of long electrical overhead transmission line spans: A modified approach. Wind and Structures, 8(2):89-106, March 2005. http://technopress.kaist.ac. $\mathrm{kr} /$ journal/container/was/was8_2_container. jsp?order=1 C876, C880, C885, C888, C890

[4] K. Anderson and P. Hagedorn. On the Energy Dissipation in Spacer-Dampers in Bundled Conductors of Overhead Transmission Lines. Journal of Sound and Vibration, 180(4):539-556, 1995. doi:10.1006/jsvi.1995.0099 C881

[5] R. B. Lehoucq and D. C. Sorensen. Deflation techniques for an implicitly restarted Arnoldi iteration. SIAM J. Matrix Anal. Appl., 17(4):789-821, 1996. doi:10.1137/S0895479895281484 C885 
[6] R. B. Lehoucq, D. C. Sorensen, and C. Yang. Solution of large-scale eigenvalue problems with implicitly restarted Arnoldi methods. ARPACK User's Guide, SIAM, 1998. http://www. ec-securehost.com/SIAM/SE06.html C885

[7] J. W. Demmel. Applied Numerical Linear Algebra. SIAM, Philadelphia, 1997. http://www.ec-securehost.com/SIAM/ot56.html C885

[8] R. J. Radke. A Matlab Implimentation of the Implicitly Restarted Arnoldi Method for Solving Large-Scale Eigenvalue Problems. PhD thesis, Department of Computational and Applied Mathematics, Rice University, Houston, Texas, 1996. http://citeseer.ist.psu.edu/radke96matlab.html C885

[9] S. S. Ranmale. Solution of transcendental eigenvalue problem in overhead conductors. Master's thesis, Department of Civil Engineering, Indian Institute of Technology Bombay, Mumbai, India, 1996. C885

[10] D. R. Fokkema, G. L. G. Sleijpen, and H. A. Van der Vorst. Jacobi-Davidson style QR and QZ algorithms for the reduction of matrix pencils. SIAM J. Sci. Comput., 20(1):94-125, 1998. doi:10.1137/S1064827596300073 C885

[11] G. L. G. Sleijpen, H. A. Van der Vorst, and Ellen Meijerink. Efficient expansion of subspaces in the Jacobi-Davidson method for standard and generalized eigenproblems. Electronic Transactions on Numerical Analysis, 7:75-89, 1998. http:

//etna.mcs.kent.edu/vol.7.1998/pp75-89.dir/pp75-89.html C885

[12] G. H. Golub and C. F. Van Loan. Matrix Computations. The Johns Hopkins University Press, London, 1989. http://www.press.jhu.edu/books/title_pages/1956.html C885 
[13] P. Hagedorn, N. Mitra, and T. Hadulla. Vortex-Excited Vibrations in Bundled Conductors: A Mathematical Model. Journal of Fluids and Structures, 16(7):843-854, 2002. 10.1006/jfls.2002.0451 C885 